\title{
Perspectives of the Interplay Between Business and Information Systems Engineering and Computer Science
}

\author{
Despite many perceived problems, the interplay between computer science \\ and business and information systems engineering (BISE) has proven extremely \\ fruitful. Important examples include data management and conceptual modeling, \\ internet-based information and transaction systems, and the emergence of compu- \\ tational business sciences. The integration of virtual and real world in the "Internet of \\ Things" offers new scientific and organizational challenges for this cooperation.
}

DOI 10.1007/s12599-008-0021-4

\section{The Author \\ Prof. Dr. Matthias Jarke \\ Lehrstuhl Informatik 5 \\ (Informationssysteme) \\ RWTH Aachen and Fraunhofer FIT \\ Ahornstr. 55 \\ 52074 Aachen \\ Germany \\ jarke@dbis.rwth-aachen.de \\ Received: 2008-09-18 \\ Accepted: 2008-10-07 \\ Accepted after one revision \\ by Prof. Dr. Buhl. \\ This article is also available in Ger- man in print and via http://www. wirtschaftsinformatik.de: Jarke $M$ (2008) Perspektiven der Wirtschafts- informatik aus Sicht der Informatik. WIRTSCHAFTSINFORMATIK. doi: 10.1007/11576-008-0127-9.}

\section{Introduction}

Informatics (in this paper used synonymously with the American term Computer Science) investigates the representation, storage, transmission and processing of information (Biundo et al. 2006). This involves the study of many different aspects, ranging from elementary data structures and processes, system principles and architectures, network communication, the design and implementation of hardware and software up to complex application systems. But computer science also reflects about the usage conditions and implications of information and communication technologies (ICT).

In Germany, informatics is nowadays understood as a combination of fundamental science and engineering science. As a fundamental science, it studies basic concepts which can be formally described and analyzed by means of mathematical formalisms and properties. These foundational studies have yielded astonishing results in the last few decades, often with equally radical effects as the spectacular developments in hardware characterized e. g. by "Moore's Law" (the doubling of processor speed every 18-24 months). For example, algorithmic advances even more than hardware acceleration have enabled the speed-up of mathematical optimization by six to seven orders of magnitude - prerequisite for better weather forecasts and realistic crash simulations. Even the continuation of "Moore's Law" into the present millennium is enabled only by multi-core architectures and therefore relies heavily on parallel algorithms. Despite numerous such successes, informatics as a foundational science is still more frequently confronted with the question of relevancy than for example mathematics.

The embedded computer systems, databases, communication networks, and multimedia interfaces of the engineering science informatics today pervade all areas of life. Around the year 2000, the number of processors has exceeded the number of humans on Earth. The omnipresence or "ubiquity" of ICT (Weiser 1993) radically changes the classical research domain of human-computer interaction. As again emphasized by the 2007 High-tech Strategy of the German Federal Government, software becomes a key tool of innovation in most business domains. In 2005, the German ICT industry has replaced the automotive industry as the business domain with the highest in-country value creation, but about half of the computer science and BISE graduates work in application industries.

Research and teaching in informatics react to these developments in two directions. Firstly, different perspectives on software technologies are being differentiated in subareas, e. g. in the Gesellschaft für Informatik e. V., GI. Software engineering, databases and information systems, human-machine interaction and IT security are evolving into cross-disciplinary themes with relevance to many different application domains. Secondly, while application-specific variants of informatics such as medical informatics or bioinformatics are well established, new specialties such as automotive informatics, cell phone informatics, or informatics in chemical engineering are either still in their infancy as branches of science, or exist in (sometimes quite happy) isolation from mainstream computer science. Many fascinating foundational questions for computer science stem from these applications. But it is a constant challenge for foundational computer science researchers to learn about them and find the right abstractions for them.

Business and information systems engineering (in this paper used interchangeably with the internationally more common term Information Systems, despite some differences in concept) has been the 
pioneer, and still is the largest representative for this interdisciplinary extension. However, the relevance of business administration for informatics goes further. Numerous scalability issues in large-scale ICT projects as well as general questions of information management (Krcmar 2004) up to the roles of Chief Information Officers (CIO) require strong business knowledge. It is therefore not surprising that business administration has been the most popular minor field of study for German informatics students for decades.

In the sequel, we first briefly discuss the evolving role of BISE between business administration, informatics, and other reference disciplines from the angle of science organizations. Subsequently, we employ the admittedly over-simplified termini of "technology push" and "application pull" to frame a discussion of the mutual interplay between Informatics and BISE. We conclude with a brief depiction of some new challenges for an even more interdisciplinary cooperation, that results from the increasing convergence and ubiquity of ICT enabled by linkage to "the real world" via sensor and actuator technologies.

\section{BISE between business admini- stration and computer science}

The relationship of the BISE / Information Systems field to its main reference disciplines has changed several times during the last decades.

In the 1980s and partly the 1990s, most BISE groups worldwide were located in business schools. Strong student demand and the dynamic development of the field led to a significant tolerance also for system-oriented, "computer sciency" types of research. For example, the CAIS department within the Stern School of Business at New York University hosted the secondlargest database research group in the US in the mid-1980s, publishing in top outlets both of the database and the (business) information systems field. Numerous other leading IS departments and research institutes e. g. in the University of Arizona, Carnegie Mellon University, Berkeley, and MIT followed similar strategies (Kambil et al. 2003). BISE in Germany used to include a fair number of system-oriented research groups as well. This is evidenced by the titles of the major focused research programs of the Ger- man national science foundation DFG, even including the more recent ones who looked at distributed systems and agentoriented applications in business.

The resulting coopetition - (some) cooperation combined with (lots of) competition - influenced the relationship between BISE and informatics for quite a while. Similar to most other areas of business administration, relatively few proposals from BISE were submitted to the DFG. Nevertheless, in compliance with general DFG policies, rejection rates of such proposals were comparable to those in other fields of science. In addition, proposers often had the feeling of "falling between the cracks", as review criteria of both reference disciplines were applied with equal severity, and genuine contributions of BISE beyond the reference disciplines were rarely understood. Despite numerous attempts, BISE has not yet succeeded to get its own subcommittee within either the Economics/Business or the informatics Standing Review Board (Fachkommission) at DFG, even though several individual candidates from BISE were nominated and sometimes elected to these boards. According to the experience of the author as an eight-year member of the computer science board, this will only change when substantially more proposals from BISE will be submitted. The often-attempted political interventions are at best promising with large-scale cooperative programs such as the above-mentioned focused research programs.

Since the mid-1990s, one can observe a stronger orientation of Information Systems towards empirical or theoretical positivist methods, and thus a certain weakening of systems-oriented IS research, at least in the US. In part, this coincides with the choice of very few "top target journals" that determine the positioning of IS departments in business school rankings and thus the tuition fee income. This trend seems to be followed by a few European countries, such as the Netherlands, and a growing number of individual economics faculties and business schools elsewhere (including Germany). In some places, e. g. the University of Karlsruhe, recently founded departments prefer the name of "Information Business" over "BISE". Nevertheless, the role of BISE within German business and economics departments currently seems to be stronger than in many North-American universities, perhaps due to the high reputation that BISE pioneers such as Peter Mertens and August-Wilhelm Scheer in Germany, or Hans Robert Hansen in Austria have achieved in both business academia and practice.

The traditional frontier between informatics and BISE thus seems to move in part into the BISE departments themselves or even to the border between BISE and business administration. In contrast, the relationship between informatics and BISE is becoming much more constructive. Nowhere is this more obvious than at the Technical University of Munich where a strong new BISE team has become part of the School of Informatics. Similarly, Scheer's Institute for Information Systems at Saarland University has joined the German Research Center for Artificial Intelligence (DFKI). A pioneer of this development was the AIFB Institute led by Wolffried Stucky that has served the Business Engineering curriculum with systemoriented IS research for several decades.

In the US, informatics-oriented IS researchers started a "Design Science" initiative around 2003 to lift the debate about BISE research to a new level (Hevner et al. 2004). The Design Science initiative revived the tradition of "The Sciences of the Artificial” (Simon 1996) and has attracted funding by the US National Science Foundation (http://science-of-design. org). Like a number of large-scale European and German national projects with strong industrial participation, this indicates a research strategy that seeks access to specific companies and individual practice innovations, but also wants to advance the necessary computer science and business foundations, within the same project. In principle, this is an interesting and promising approach. However, the sheer size of some of the resulting projects with many partners of different interests leads to risks of failure which should not be underestimated.

\section{Technology push and application pull: three examples of successful interplay between computer science and BISE}

In his bestselling book "The World is Flat", Thomas Friedman (2005) argues convincingly that standards and platform strategies from communications engineering and computing have been the main drivers for successful applications 
and business innovations. Already in the mid-1980s, August-Wilhelm Scheer argued for an information processing-oriented approach to business analysis that includes such developments stringently in all business theories and models of business administration research (Scheer 1984).

Close to hardware, the "Wintel" de facto standards for PC platforms and operating systems have to be mentioned. At least equally impressive is the development of mobile telephony following the (almost) worldwide acceptance of the GSM standard in the mid-1990s. However, the adoption problems of the follow-up 3G standard UMTS showed equally convincingly that platforms alone are not enough. The working group Next-Generation Mobile Networks (NGMN) of the Telecom industries therefore attempts to establish application innovation in parallel to, or even as a driver for technology standardization.

Nevertheless, the most important successes of the BISE/Information Systems field concerning design science can be found in the modeling sector around complex software-intensive systems. Here, it is of decisive importance that computer science abstractions are available that radically simplify complex real-world phenomena and thus make them amenable to formal analysis and optimization. The basics of these theories must be sufficiently easy-to-use, such that domain specialists can employ them to develop complex applications. Yet, the complexity of these applications almost always creates new technical and formal challenges for new theoretical developments. In this section, we choose three important examples of this interplay between informatics and BISE, from which perhaps some considerations for future research strategies can be derived.

\subsection{Data management and conceptual modeling}

Undoubtedly, the relational data model (Codd 1970) has been one of the most important computer science theories for business. It reduced the complex question of administrative data management conceptually to the handling of real and virtual tables. Algebra-based relational query languages offered the important closure property that the answer to a query over a set of tables is a table again, and can thus be easily reused in further database operations. The significant complexity of making this simple model work efficiently is nowadays hidden in relational DBMS products that took over a decade to develop. This did not only create a multi-billion dollar industry of DBMS vendors such as Oracle, Microsoft, IBM, Sybase, etc. More importantly, Enterprise Resource Planning (ERP) systems such as SAP R/2 or SAP R/3 could not have been built without this background technology. However, the domain-related complexity of up to a five-figure number of tables with thousands of parallel users resulting from such BISE applications continuously challenged the limits of relational technologies and offered new challenges to computer science, including high-performance transaction management, query optimization, high-availability systems, and energy optimization.

Conceptual modeling demonstrates the interplay of seemingly simple computer science abstractions and the richness of the BISE domain equally impressively. In its original form, the Entity-Relationship Model (Chen 1976) contained exactly these two basic concepts, plus a simple idea of attributes and roles. Empirical studies have indeed shown again and again that users and analysts are rarely able to handle a larger number of base constructs consistently. However, the presence of thousands of product and process variants in business quickly leads to models of extreme complexity to which BISE research has reacted with genuine transdisciplinary innovations such as domain-specific reference models (Scheer 2002). These reference models do not just address data modeling, but also business process modeling, and meanwhile also cover boundaryspanning concepts such as supply chain management and customer relationship management. Here, we see fundamental BISE research, but also new challenges for computer science related to the organization and integration of very large data and process models, e. g. via metamodeling (Mylopoulos et al. 1990). Manifold interdisciplinary research issues also exist at the boundary between conceptual process modeling and its implementation in different kinds of workflow systems (v. d. Aalst and Kumar 2003).

\subsection{Standards and platform-strategies in the internet}

The Internet has been one of the main influencing factors on business development during the last decade. Together with data management and process modeling, its first-generation manifestations such as email enabled the business process reengineering movement since the late 1980s and the radical flattening of management hierarchies by larger communication and control spans.

However, only the introduction of highperformance fibre - the "Information Highway" propagated by the Clinton/ Gore administration in the early 1990s formed the infrastructure for the present developments, which are characterized by eight-digit active user numbers, self-organization, and full usage of media in communication. For business innovation planning, the right timing has proven decisive. Even excellent ideas can fail despite huge investments or vanish as dinosaurs from the market, if they move too far ahead of technical standards. Conversely, the adoption of such standards creates an ecology for innovation which is usually filled extremely quickly, because in computing development costs may be high but marginal production costs are extremely low.

Friedman's (Friedman 2005) observation that the Internet standards evolve systematically from bottom to top, without the chance of skipping a step in between, is therefore of equally high importance for computer science and BISE. More specifically:

- Since 1993, the http-Protocol by Tim Berners-Lee enabled the explosive growth of the Internet usage by the World Wide Web, initially as a publication medium. The GSM protocol drove the number of mobile phones to over one third of the world population (3.1 bn in early 2008) within the same 15 years.

- The first electronic commerce platforms from BISE contributed significantly to the development of web server technologies. However, open source solutions such as Apache made it possible for millions of small businesses and citizens to develop their own web servers and paved the way for today's Social Web (or Web 2.0). The chances for innovation resulting from this proliferation of decentralized initiatives of course also caused some loss of control 
in larger enterprises. The big vendors have begun to address these issues by complex platforms such as NetWeaver, but they are far from solved yet.

- The standardization of the basic web service and data exchange protocols SOAP and XML provides the foundation for workflow standards and platforms, that orchestrate service execution and information exchange across technical platforms, at least at a syntactic level.

- With the convergence of web services and mobile telephony finally reaching the breakthrough also for the $3 \mathrm{G}$ standards like UMTS through products like the iPhone, new opportunities for innovation are opened up. However, this step also increases the long-neglected problems of energy consumption and $\mathrm{CO} 2$ emissions.

Each of these evolutionary steps initially looks like a typical Technology Push example. However, this is always followed very quickly by BISE innovations, such as eCommerce around the year 2000, Business Process Management and Enterprise Application Integration a few years later. In turn, these business strategy innovations create follow-up research problems that are inherently interdisciplinary not just between informatics and BISE, but also with Legal and Social Sciences. For example, the eBusiness sector has radically changed the problems of security and privacy, for which the isolated solutions of the past do not work anymore. But it also created completely new challenges to reliability, performance and scalability of question-answering systems (search engines with sometimes millions of parallel users) and business transaction handling. Last but not least, the huge amount of transaction log data produced by these systems permit computer-assisted empirical business research, and thus transforms several sectors of business administration research into Computational Sciences.

\subsection{BISE as a computational science}

It seems interesting to transfer the recent idea of the so-called Computational Sciences to the hypothetical concept of a "computational business science". According to Jim Grays' (Gray et al. 2005) pioneering work, most sciences have followed a method schema whose last two steps have only become possible by recent advances in computer science:
1. Empirical observations of reality followed by first experiments

2. Development of initial precise theories (often formalized mathematically or by other means), associated with targeted attempts for falsification as a means of scientific progress

3. Computer-assisted simulation or data mining with optimized visualization of results as a third methodological variant in addition to experiment and theory

4. Distributed and cooperative data-centric analysis on the basis of worldwide shared platforms of more or less public base data. Jim Gray pioneered this within Microsoft Research and attracted almost a million users to his huge distributed astronomy data set, almost one hundred times the number of professional astronomers in the world.

Simulation and data mining on the basis of very large data sets presuppose the availability of high performance computing as well as efficient algorithms adapted to the application domain. Again, we find a long tradition of cooperation between computer science and BISE research in this area. It was the BISE field that pointed out already in the beginning of the 1980s that rule-based expert systems in business applications can only be successful if they are closely linked to internal business data or external data sources. Success stories for such methods can be found, for example, in Computational Finance and in Customer Relationship Management.

One is reminded of stage 4 above, the data-centric approach to computational sciences, when all market participants share more or less the same base information (e. g. stock value developments). In business, such a situation is not necessarily as positive as in the natural sciences where discoveries make you famous and help others, as it makes newly detected business patterns very quickly obsolete and thus continuously increases the informatics requirements in the worldwide competition (Dhar 1998). In grid computing, worldwide distributed computational resources of different owners are temporarily linked in order to solve extremely complex parallel computing problems. These new hardware and software technologies, initially considered for the natural sciences and biomedicine, are nowadays not just used in compute-intensive business problem solving. Indeed, they have led to the genuine BISE innovation of transforming the virtualization of computing resources into a business strategy for reduction of cost and environmental impact. Once more, this illustrates how theories from BISE reflect back to technological requirements and create interdisciplinary research needs that reach to the foundations of both disciplines.

\section{Co-evolution: chances of transdisciplinary research and innovation}

The mentioned examples are but a few cases of fruitful interchange between informatics and BISE, both concerning the research questions and the innovative results and practice solutions. For example, the emergence of empirical software engineering research goes largely back to problem analyses and approaches of early BISE, but of course also to shared roots with pioneers like Frederic Brooks (1987) who were both successful computer scientists and successful managers. Questions of Information Management were also first defined in BISE and only afterwards considered in their technical implications. But successful solutions require input from both sides. Topics such as Outsourcing and Offshoring should also be mentioned in this context.

The interplay of informatics and BISE is currently reaching an interesting point. The convergence of technologies within the virtual world - data management, service-oriented programming, internet and mobile communication - is slowly reaching its natural end. Even though much remains to be researched (Jarke 2008), the next steps could be much more radical. The further growing numbers of processors and subsystems are not just networked among each other, but interact via sensors and actuators directly with the "real world". RFID radio chips constitute a first major step towards the mobile "Internet of Things" (Bullinger and ten Hompel 2007; Floerkemeieret al. 2008), that already massively changes business aspects including logistics, sales, and complaint management. It is promising to see that, in this domain, the cooperation does not just include computer scientists and people from BISE but also engineers and lawyers. Even more than in the three examples described in the last section, the hard fundamental questions in 


\section{Abstract \\ Matthias Jarke \\ Perspectives of the Interplay Between Business and Information Systems Engineering and Computer Science}

The relationship between business and information systems engineering (BISE) and its main reference disciplines business administration and informatics has changed several times during the last decades. Despite some frictions during the 1980s and 1990s, the interplay between informatics and BISE is becoming much more constructive and has proven extremely fruitful. Important examples for this co-evolution include data management and conceptual modeling, internet-based information and transaction systems, and the emergence of computational business sciences. The increasing integration of virtual and real world in the "Internet of Things" offers new scientific and organizational challenges for the cooperation between BISE and informatics, which can only be formulated and solved jointly.

Keywords: Computer science, Business and information systems engineering, Co-evolution, Interdisciplinary research this area can only jointly be formulated, let alone be solved. University training and continuing education will have to adapt to this increasingly networked situation, in order to remain successful in the international competition.

\section{Acknowledgment}

This work was supported in part by the Deutsche Forschungsgemeinschaft DFG in its Excellence Cluster "Ultra-Highspeed Mobile Information and Communication" at RWTH Aachen University.

\section{References}

Biundo S, Claus V, Mayr HC (2006) Was ist Informatik? Gesellschaft für Informatik e. V.

Brooks FP (1987) No silver bullet: essence and accidents of software engineering. IEEE Computer 20(4):10-19

Bullinger HJ, ten Hompel M (2007) Internet der Dinge. Springer, Heidelberg

Chen P (1976) The entity-relationship model: toward a unified model of data. ACM Transactions on Database Systems 1(1):9-36

Codd EF (1970) A relational model of data for large shared data banks. Comm ACM 13(6):377387

Dhar V (1998) Data mining in finance: using counterfactuals to generate knowledge from organizational information systems. Information Systems 23(7):423-437

Floerkemeier C, Langheinrich M, Fleisch E, Mattern F, Sarma SE (eds) (2008) The internet of things. Proc. first intl conf IOT 2008, Zürich. LNCS 4952, Springer, Heidelberg

Friedman TL (2005) The world is flat. Penguin, London

Gray J, Liu DT, Nieot-Satisteban MA, Szalay AS, DeWitt DJ, Heber G (2005) Scientific data management in the coming decade. ACM SIGMOD Record 34(4):34-41

Hevner AR, March ST, Park J, Ram S (2004) Design science in information systems research. MIS Quarterly 28(1):75-105

Jarke $M$ (2008) Holistic engineering of ultra-highspeed mobile information and communication systems. In: Kaschek R, Kop C, Steinberger C, Fliedl G (eds) Information systems and ebusiness technologies, proc 2 nd intl conf UNISCON 2008, Klagenfurt. LNBIP 5, Springer, Heidelberg, pp 148-157

Kambil A, Weill P, Jarke M, Filos E, Loebbecke C, Feeny D (2003) Different IS research communities: are they competitors, complements, or ignoring each other. Comm AIS 11:513-524

Krcmar H (2004) Informationsmanagement, 4th edn. Springer, Heidelberg (english version in press)

Mylopoulos J, Borgida A, Jarke M, Koubarakis M (1990) Telos - representing knowledge about information systems. ACM trans information systems 8(4):325-362

National Science Foundation (oJ) Science of design - software-intensive systems. http:// www.nsf.gov/pubs/2004/nsf04552/nsf04552. $\mathrm{htm}$

Scheer AW (1984) EDV-orientierte Betriebswirtschaftslehre. Springer, Heidelberg

Scheer AW (2002) Business process engineering. Reference models for industrial enterprises, 2nd edn. Springer, Heidelberg

Simon HA (1996) The sciences of the artificial, 3rd edn. MIT Press, Cambridge

v. d. Aalst WMP, Kumar A (2003) XML-based schema definition for support of inter-organizational workflow. Information Systems Research 14(1)

Weiser M (1993) Some computer science issues in ubiquitous computing. Comm ACM 36(7):7484 